\title{
Knowledge and Practice of Physicians to Hyperglycemia in Pregnancy: A Nationwide Survey in Bangladesh
}

\author{
Shahjada Selim ${ }^{1}$, Rezaul Karim Kazal ${ }^{2}$, ABM Kamrul-Hasan ${ }^{3, \text { * }}$ \\ ${ }^{1}$ Department of Endocrinology, Bangabandhu Sheikh Mujib Medical University, Dhaka, Bangladesh \\ ${ }^{2}$ Department of Obstetrics \& Gynaecology, Bangabandhu Sheikh Mujib Medical University, Dhaka, Bangladesh \\ ${ }^{3}$ Department of Endocrinology, Mymensingh Medical College, Mymensingh, Bangladesh \\ Email address: \\ selimshahjada@gmail.com (S. Selim),rkkazal@yahoo.com (R. K. Kazal), rangassmc@gmail.com (ABM Kamrul-Hasan) \\ ${ }^{*}$ Corresponding author
}

\section{To cite this article:}

Shahjada Selim, Rezaul Karim Kazal, ABM Kamrul-Hasan. Knowledge and Practice of Physicians to Hyperglycemia in Pregnancy: A Nationwide Survey in Bangladesh. International Journal of Diabetes and Endocrinology. Vol. 5, No. 4, 2020, pp. 83-88.

doi: $10.11648 /$ j.ijde.20200504.16

Received: December 3, 2020; Accepted: December 16, 2020; Published: December 25, 2020

\begin{abstract}
The frequency of hyperglycemia in pregnancy is high in Bangladesh, but very little is known about the current knowledge and practice about hyperglycemia in pregnancy in our practicing doctors. The objective of the present study was to assess the knowledge and practice of the doctors across the country who are involved in the management of hyperglycemia in pregnancy. Structured pretested data collection sheets were sent to the doctors taking care of hyperglycemia in pregnancy (endocrinologists, obstetricians, and others) through email and postage. The questionnaire focused on their knowledge and practice pattern on hyperglycemia in pregnancy. In total, 950 doctors of Bangladesh were invited to join the survey, and 262 (24.89\%) responded. Among the participants, $42.3 \%$ (111) were endocrinologists, $27.10 \%$ (71) obstetrician/gynecologist, and $30.53 \%$ (80) were other physicians, including internists, diabetes family physicians. The majority of the participants were aware of hyperglycemia's adverse outcomes in pregnancy, advocates universal screening, and followed the American Diabetes Association's recommendations for the screening purpose. Though most of them use insulin to manage hyperglycemia in pregnancy, more than half prefer to continue metformin if used for other causes. The majority of the responders favored the Caesarian section as the preferred delivery method. The study participants admit that more than half of the gestational diabetes cases are lost to follow up after delivery. This study's findings may serve as a baseline for the management of hyperglycemia in pregnancy in this country. The doctors involved in managing hyperglycemia in pregnancy require more training for knowledge building and rational practice.
\end{abstract}

Keywords: Hyperglycemia in Pregnancy, Gestational Diabetes Mellitus, Knowledge and Practice Pattern, Bangladesh

\section{Introduction}

The prevalence of hyperglycemia in pregnancy is increasing, along with the upward trends of type 2 diabetes mellitus; according to the International Diabetes Federation (IDF), the global prevalence is $15.8 \%$, but it is $27.0 \%$ in South East Asian (SEA) countries [1]. Hyperglycemia in pregnancy may be pre-gestational or gestational (gestational diabetes mellitus, GDM); both types pose serious health consequences for the affected mother and the offspring both in the short and long-term [2,3]. Appropriate treatment of maternal hyperglycemia reduces such risks almost to the level seen in normoglycemic pregnant women [4]. The importance of recognizing and managing hyperglycemia in pregnancy is now well-accepted, but there are still no universally accepted criteria for screening and diagnosing this condition. Despite several organizations' guidelines, controversy exists on the optimal screening strategies and diagnostic criteria for GDM [5]. The management approach for GDM also varies greatly; though lifestyle modification is universally suggested for such patients, pharmacological management is not uniform. All these might have implications on the prevalence of hyperglycemia in pregnancy, maternal, fetal, and postnatal outcomes [5].

In Bangladesh, various healthcare professionals (HCPs), 
including endocrinologists, internists, obstetricians, general physicians, and others, manage hyperglycemia in pregnancy. Data on the diagnostic and management approach of hyperglycemia in pregnancy lack here. The objective of this study was to assess the current practices of the doctors managing hyperglycemia in pregnancy in Bangladesh. The results are anticipated to draw increased attention to their potential but also their limitations in GDM management.

\section{Materials and Methods}

We conducted this survey from November 2017 to March 2018 among the physicians (endocrinologists, obstetricians, and others) all over Bangladesh who are taking care of the women having hyperglycemia in pregnancy. A structured pretested questionnaire focusing on their knowledge and practice pattern on hyperglycemia in pregnancy has been sent through email and postage along with instructions to fill that up. The questionnaire was developed by extensive reviewing of the available literature. It was in English, given the high level of English fluency among the target physicians. The questionnaire addressed their practice of using the screening methods to diagnose hyperglycemia in pregnancy, management patterns, including lifestyle, pharmacotherapy and glycemic monitoring, perinatal care, and postpartum follow-up. The questionnaire was sent to 950 physicians, the procedure and purpose of the study were described in detail, and they were asked to fill the consent form before participating in this study. The introduction to the questionnaire made it clear that the survey was anonymous and confidential. Physicians responded to statements regarding management of hyperglycemia in pregnancy on a three-point Likert-scale (always, sometimes, never/not frequently). The drug use pattern included options of available insulins preparations, Glibenclamide, and Metformin; the respondents could choose more than one option. The options for the preferred time of delivery were either before 38 weeks or beyond 38 weeks of gestation, and the preferred procedure of delivery was either vaginal delivery or Caesarian section. There were some open questions for the assessment of some parameters. The questionnaire was designed to be completed in 15 minutes. A reminder was sent by email to the physicians after one month of the initial contact to obtain a high response rate. The investigators examined all feedback for completeness and discrepancy of data; any incomplete or multiple feedback was discarded. All data were descriptively analyzed by using Microsoft Excel, version 2019 (Microsoft Corporation, 2019).

\section{Results}

Out of 950 doctors invited to join the survey, 262 (24.89\%) responded. The survey covered eight divisions of Bangladesh. Among the respondents, $42.4 \%$ (111) were endocrinologists, $27.1 \%$ (71) obstetrician/gynecologist, and 30.5\% (80) were other physicians (internists, diabetes family physicians, etc.). Almost all (94.7\%) of them were aware of the high prevalence of hyperglycemia in pregnancy in the country.

The majority $(65.6 \%)$ of the responders stated that all pregnant women should be tested (i.e., universal screening), and the rest, $34.4 \%$ opined that only women with risk factors should be screened. $79.0 \%$ of them favored the early screening to prevent complications of hyperglycemia in pregnancy. $88.2 \%$ also stated that mild glucose intolerant (mild GDM) women in the first trimester should be retested at 20-24 weeks of gestation. Three-fourth (75.6\%) admits the usefulness of $\mathrm{HbAlc}$ in differentiating GDM and pre-gestational DM.

For screening of hyperglycemia in pregnancy, more than half $(53.4 \%)$ used the OGTT-criteria suggested by the American Diabetes Association (ADA), 31.3\% used fasting plasma glucose (FPG) and plasma glucose 2-hours after breakfast (PG-2HABF), and 9.5\% used the World Health Organization (WHO) criteria (Figure 1).

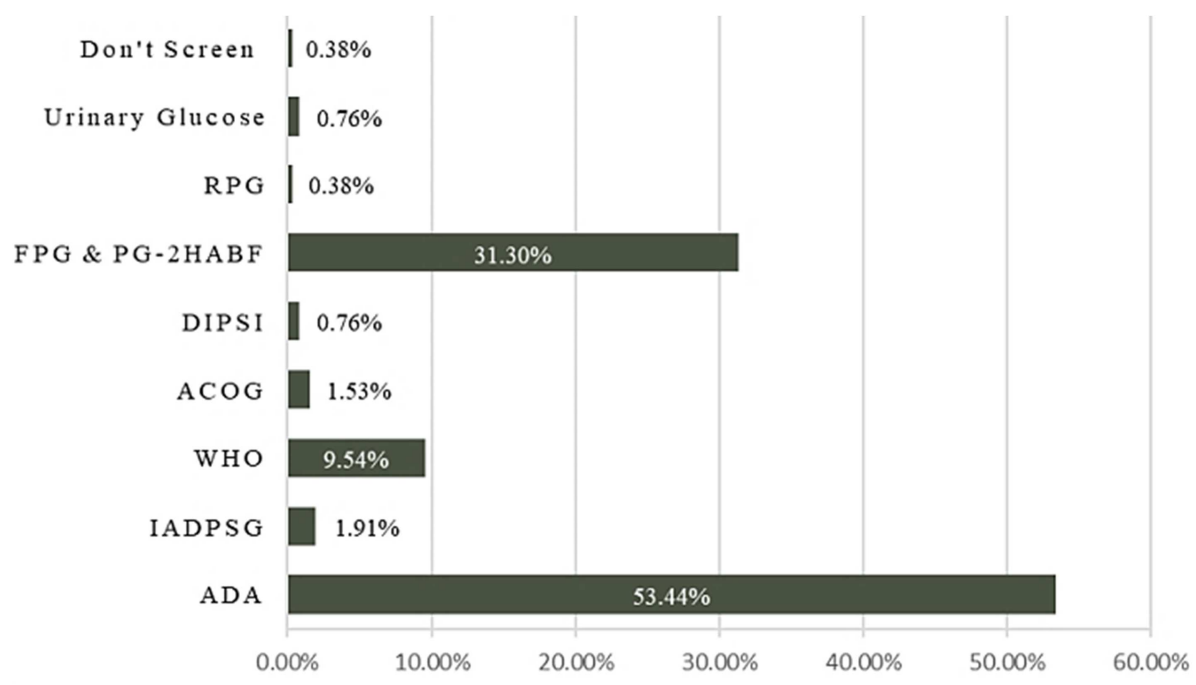

$[\mathrm{RPG}=$ Random plasma glucose; $\mathrm{FPG}=$ Fasting plasma glucose; $\mathrm{PG}-2 \mathrm{HABF}=$ Plasma glucose 2-hours after breakfast $]$

Figure 1. Screening Tests Used by the Study Participants for Diagnosis of Hyperglycemia in Pregnancy. 


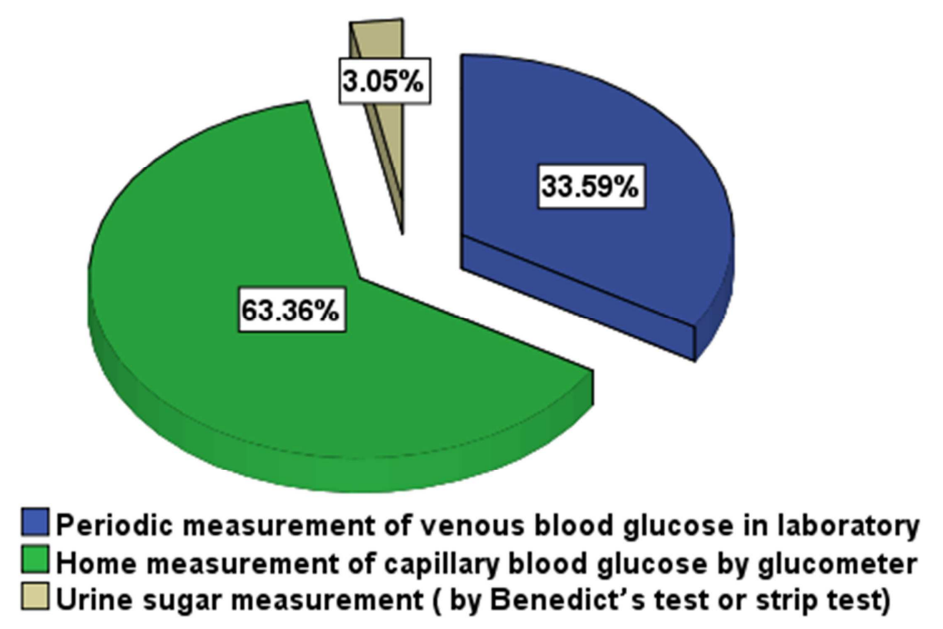

Figure 2. Strategy for monitoring blood glucose in pregnancy.

Table 1. The pattern of hyperglycemia in pregnancy management by the respondents.

Counsel for diet appropriate for GDM

Advice for physical activity suitable for pregnancy

Ask support from nutritionists or dieticians for the management of GDM

Involve family members and caregivers during counseling of GDM women

Preferred drug as first-line treatment of GDM

Usual frequency of prescribed drug in GDM

Continue metformin during pregnancy if the patients were on it before the pregnancy due to any other cause (e.g., PCOS)

Prescribe oral drugs when patients refuge or unwilling to take insulin

Allow artificial sweeteners in pregnancy

Try to achieve and maintain the blood glucose target throughout the pregnancy

$\begin{array}{ll}\text { Always } & 88.6 \% \\ \text { Sometimes } & 8.8 \% \\ \text { Never } & 2.6 \% \\ \text { Always } & 54.6 \% \\ \text { Sometimes } & 45.4 \% \\ \text { Always } & 61.8 \% \\ \text { Sometimes } & 37.8 \% \\ \text { Never } & 0.4 \% \\ \text { Always } & 75.9 \% \\ \text { Sometimes } & 21.8 \% \\ \text { Never } & 2.3 \% \\ \text { Insulin } & 86.6 \% \\ \text { Metformin } & 13.4 \% \\ \text { Glibenclamide } & 0 \% \\ \text { Metformin } & 7.7 \% \\ \text { Insulin } & 65.5 \% \\ \text { Premixed Insulin } & 12.1 \% \\ \text { Split mixed Insulin } & 13.8 \% \\ \text { Basal Bolus } & 17.2 \% \\ \text { Bolus Insulin Only } & 10.1 \% \\ \text { Glibenclamide } & 0.1 \% \\ \text { Always } & 26.7 \% \\ \text { Sometimes } & 34.0 \% \\ \text { Never } & 39.1 \% \\ \text { Always } & 13.7 \% \\ \text { Sometimes } & 45.1 \% \\ \text { Never } & 41.2 \% \\ & 42.7 \% \\ \text { Always } & 73.3 \% \\ \text { Sometimes } & 23.7 \% \\ \text { Not frequently } & 3.0 \% \\ & \\ & \\ & \\ & \end{array}$

Table 2. The pattern of delivery and postpartum care by the respondents.

\begin{tabular}{|c|c|c|}
\hline \multirow{3}{*}{ Considers that the GDM women may require special care for delivery and postnatal period } & Always & $93.1 \%$ \\
\hline & Sometimes & $6.1 \%$ \\
\hline & Never & $0.8 \%$ \\
\hline \multirow{2}{*}{ Preferred time of delivery } & Before 38 weeks & $67.2 \%$ \\
\hline & Beyond 38 weeks & $29.4 \%$ \\
\hline \multirow{2}{*}{ The preferred procedure of delivery } & Vaginal delivery & $21.8 \%$ \\
\hline & LUCS & $78.2 \%$ \\
\hline Frequency of GDM patients followed up for persistence of high blood glucose at postpartum & & $46.2 \%$ \\
\hline
\end{tabular}




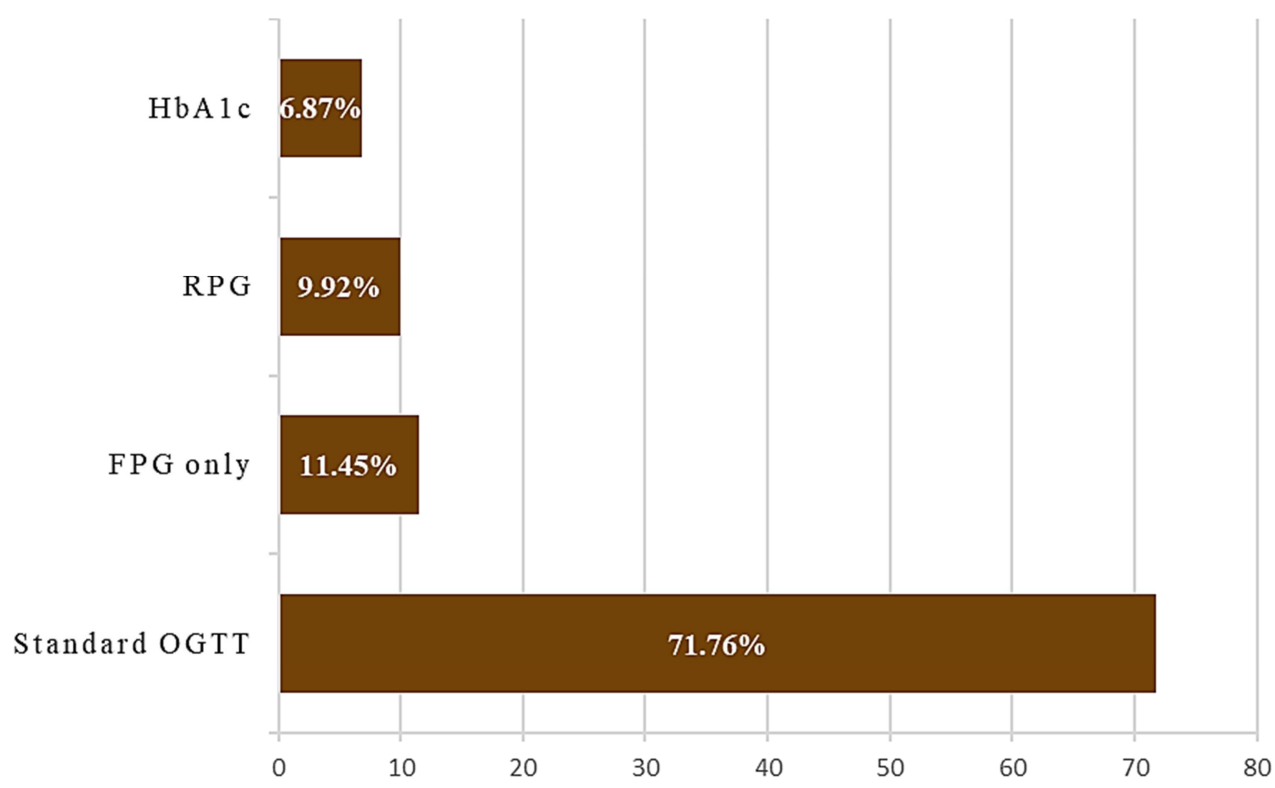

$[\mathrm{RPG}=$ Random plasma glucose; $\mathrm{FPG}=$ Fasting plasma glucose; OGTT $=$ Oral glucose tolerance test $]$

Figure 3. Tests done for follow up for persistence of high blood glucose at postpartum.

\section{Discussion}

The screening and diagnostic criteria for hyperglycemia in pregnancy, especially GDM, are still in debate, and there is discordance in using those methods by different physicians. Once identified, those women can consider further tests, and health care providers can offer interventions that may improve their health and that of their infant. For GDM, the oral glucose tolerance test (OGTT) is generally the diagnostic test of choice; this test is usually administered at the first antenatal visit and between 24 and 28 weeks of gestation if the first test is normal [2]. The American College of Obstetricians and Gynecologists (ACOG) and United Kingdom (UK) National Institute for Health and Care Excellence (NICE) recommended that women who have had GDM in a previous pregnancy should be offered diagnostic testing as early as possible after pregnancy booking (in either the first or second trimester) to identify undiagnosed type 2 diabetes [6, 7]. ACOG also recommends early testing in obese women or women with impaired glucose metabolism [6]. The Australasian Diabetes in Pregnancy Society (ADIPS) recommends risk factor assessment in early pregnancy; risk factors are ranked by severity, and either one "high" risk factor or two "moderate" risk factors are needed before an OGTT is offered [8]. Compared with the selective screening strategy practiced in most western countries, universal screening for GDM detects more cases and improves maternal and neonatal prognosis. In the Indian subcontinent context, screening is essential in all pregnant women as women in this area have an 11-fold increased risk of developing glucose intolerance during pregnancy compared to Caucasian women. On this background, The Diabetes In Pregnancy Study group India (DIPSI) recommends universal screening to detect hyperglycemia in pregnancy [9]. However, this practice is not uniform in this area. In India, Mahalakshmi et al. found that $84.9 \%$ of obstetricians and gynecologists screened all pregnant women for GDM, while $14.5 \%$ preferred to do only risk-based screening [10]. In another study conducted by Appajigol et al. in the same country, only $22 \%$ of family physicians and $58.3 \%$ of obstetricians practiced universal screening for GDM [11]. About two-thirds (65.6\%) of the responders favored universal screening in the current study. In Morocco, a similar number of healthcare providers (69.9\%) advocated universal screening for GDM [12]. According to Carballo et al., the vast majority (96.2\%) of the health professionals from the primary health care (PHC) centers in Kuwait felt that pregnant women should routinely be screened for GDM [13].

Although controversy exists, most of the recommending authorities suggest that women with risk factors and high suspicion of undiagnosed type 2 DM (i.e., obesity, metabolic syndrome) should be screened before pregnancy or at the first prenatal visit (early screening). In the absence of early screening or for women negative to early screen, universal screening should be performed at 24 to 28 weeks [2, 4, 6, 7, 9, 14]. In this study, $79.0 \%$ of the physicians favored the early screening to prevent complications of hyperglycemia in pregnancy; $88.2 \%$ also stated that mild glucose intolerant (mild GDM) women in the first trimester should be retested at 20-24 weeks of gestation. Mahalakshmi et al. observed that most $(67.8 \%)$ of the Indian obstetricians and gynecologists performed screening for GDM in the first trimester; the screening was performed between 20 and 28 weeks by $40 \%$ and after 28 weeks by $2.8 \%$ [10]. Among the Moroccan health professional, $53.3 \%$ opined that GDM detection should occur in the second trimester, and only $30.4 \%$ stated screening is indicated in the first trimester of the pregnancy or at the first contact with the woman [12]. Most (82\%) of the PHC staff in Kuwait said that women should be screened at the first 
antenatal visit; only $32.7 \%$ of respondents said that women are screened between the 24 th-28th week, and $13.5 \%$ referred to GDM screening after 28 weeks [13].

Screening tests for GDM is somewhat controversial and can be performed either with a One-Step or with a Two-Step approach. Recommendations of international organizations are not standardized [15]. There is no national guideline for screening for GDM in Bangladesh, but most of the endocrinologists follow the ADA criteria [2]. In this study, $53.44 \%$ of the physicians screened pregnant women following the same. WHO, ACOG, and DIPSI criteria were followed by $9.54 \%, 1.53 \%$, and $0.76 \%$ of the respondents. The neighboring country India, has the national guidelines for it, and DIPSI criteria are frequently used there [9-11]. One study from India found $78.3 \%$ of the family physicians and obstetricians followed the national guideline in screening GDM [11]. In another study conducted by Mahalakshmi et al., $36.7 \%$ of the responders reported using the DIPSI criteria, $24.7 \%$ the World Health Organization (WHO) 1999 criteria, 23.8\% the International Association for Diabetes and Pregnancy Study Groups (IADPSG) criteria, and $14.8 \%$ the ADA 2-step method. The picture was not the same among the physicians/diabetologists/endocrinologists in the same study; among whom $29.4 \%$ reported using the DIPSI criteria, $22.5 \%$ the WHO 1999 criteria, 19.1\% the IADPSG criteria, 29\% the ADA criteria [10]. In Kuwait, the WHO recommended method for screening emerged as the most common technique (72.1\%), while $22.1 \%$ referred to the classic diagnostic test, and $4.8 \%$ referred to "other" techniques [13]. Almost one-third (31.3\%) of the physicians who responded in this survey did fasting plasma glucose (FPG) and plasma glucose 2-hours after breakfast (PG-2HABF) to detect GDM here, which did not comply with any recommendations. Notably, $65.6 \%$ of the respondents favored universal screening for GDM in Bangladesh though there were not sure about the appropriate screening test for GDM. More interestingly, $75.6 \%$ of the physicians preferred $\mathrm{HbAlc}$ to differentiate GDM and pre-gestational DM in this survey. In Belgium, the most used screening tests for pregestational diabetes were the measurement of a fasting plasma glucose (FPG) $(35 \%)$ or a random glycemia (35\%); HbAlc was used in $14 \%$ instances [16]. Only a few $(0.76 \%)$ of the respondents of this study used urinary glucose testing for diagnosing hyperglycemia in pregnancy. $38.5 \%$ of the doctors used urine glucose to diagnose GDM in Morocco, according to the observation of Utz et al. [12]. Mahalakshmi et al. observed that $0.6 \%$ of the doctors in India did not screen for GDM in pregnant women; the frequency is $0.38 \%$ in the current survey [10].

The use of antidiabetic drugs in pregnancy is not uniform in Bangladesh like in other countries. The majority $(86.6 \%)$ of the respondents in this survey prefer insulin for managing hyperglycemia in pregnancy, metformin is preferred by $13.4 \%$, and none prefers glibenclamide. ADA recommends only insulin to manage hyperglycemia in pregnancy as oral antidiabetic drugs lack long term safety data [2]. NICE permits the use of metformin and glibenclamide to use in this condition [7]. Ununiform use of antidiabetic drugs to manage hyperglycemia in pregnancy is also observed in India. Mahalakshmi et al. observed that $50.4 \%$ of diabetologists/endocrinologists used insulin for all women with GDM, 37.5\% used insulin only for some, and $12.1 \%$ reported using no insulin at all when drug was needed to manage GDM. Among those who preferred using oral hypoglycemic agents, metformin was used by $53.8 \%$, sulfonylureas by $3.6 \%$, and a combination of the two by $12.2 \%$ [10]. In the same country, Appajigol et al. found only $38.9 \%$ of family physicians were using insulin to treat GDM, whereas $75 \%$ of obstetricians using insulin [11].

As nearly $50 \%$ of these women with GDM develop T2DM within 5-10 years after delivery, it is crucial to check their glucose levels postpartum [2]. Thus, regular screening for T2DM should be strongly encouraged. Many organizations have issued guidelines for postpartum follow-up of women with GDM [2, 6-9]. The ADA recommends that women with GDM should undergo screening for T2DM with OGTT six weeks after delivery [2]. According to the current survey, only $46.2 \%$ of the GDM cases are tested postpartum for persistence of hyperglycemia. In India, according to Mahalakshmi et al.'s study, $56 \%$ of physicians/diabetologists/endocrinologists and $71.6 \%$ of obstetricians and gynecologists said they advised women with GDM to undergo oral glucose tolerance testing (OGTT) after delivery [10]. In the current survey, $71.76 \%$ of respondents used formal OGTT for postpartum evaluation of GDM, fasting plasma glucose $(11.45 \%)$, random plasma glucose $(9.92 \%)$, and the others used HbAlc (6.87\%). In Belgium, Fasting glycemia was the preferred test for postpartum follow-up [17]. Overall, the OGTT was the preferred test (57\%) among all doctors in England, although general practitioners preferred fasting glucose $(50 \%)$ and glycated hemoglobin (47\%) [18].

Screening and diagnosis of GDM and treating it prevent adverse maternal and perinatal outcomes and future diabetes in both mother and child. Whatever method is used, it is essential to do universal screening in Southeast Asian countries, including Bangladesh. More effective and simpler strategies should be developed for future clinical practice. The most robust guidelines for screening of GDM from the relevant authority is yet to come [19, 20].

As the multi-disciplinary approach of GDM management has undoubtedly proven useful in predicting and achieving a better outcome, all concerned GDM-care physicians should be brought to a unified clinical approach to it.

\section{Conclusion}

Many of the physicians are aware of the adverse outcomes of hyperglycemia in pregnancy and advocate universal screening. They usually follow the ADA guideline to diagnose and manage GDM. Most of the concerned physicians initiate insulin, but more than half prefer to continue metformin if used for other causes. About half of the GDM mothers were lost to follow up after delivery. As is the pioneer study in Bangladesh to determine the approach to GDM of the responding physicians, this study's finding may 
serve as a baseline for managing hyperglycemia in pregnancy. We also emphasize that the doctors involved in managing hyperglycemia in pregnancy require more training for knowledge building and improvement of rational practice.

\section{Conflicts of Interest}

There are no conflicts of interest.

\section{References}

[1] International Diabetes Federation (2019). IDF Diabetes Atlas, 9th ed. Brussels, Belgium: International Diabetes Federation. Available at: http://www.diabetesatlas.org.

[2] American Diabetes Association (2020). 2. Classification and Diagnosis of Diabetes: Standards of Medical Care in Diabetes-2020. Diabetes Care, 43 (Suppl 1), S14-S31.

[3] HAPO Study Cooperative Research Group, Metzger, B. E., Lowe, L. P., Dyer, A. R., Trimble, E. R., Chaovarindr, U., Coustan, D. R., Hadden, D. R., McCance, D. R., Hod, M., McIntyre, H. D., Oats, J. J., Persson, B., Rogers, M. S., \& Sacks, D. A. (2008). Hyperglycemia and adverse pregnancy outcomes The New England Journal of Medicine, 358 (19), 1991-2002.

[4] Crowther, C. A., Hiller, J. E., Moss, J. R., McPhee, A. J., Jeffries, W. S., Robinson, J. S., \& Australian Carbohydrate Intolerance Study in Pregnant Women (ACHOIS) Trial Group (2005). Effect of treatment of gestational diabetes mellitus on pregnancy outcomes. The New England Journal of Medicine, 352 (24), 2477-2486.

[5] Mohan, V., Mahalakshmi, M. M., Bhavadharini, B., Maheswari, K., Kalaiyarasi, G., Anjana, R. M., Uma, R., Usha, S., Deepa, M., Unnikrishnan, R., Pastakia, S. D., Malanda, B., Belton, A., \& Kayal, A. (2014). Comparison of screening for gestational diabetes mellitus by oral glucose tolerance tests done in the non-fasting (random) and fasting states. Acta Diabetologica, 51 (6), 1007-1013.

[6] Committee on Practice Bulletins-Obstetrics (2013). Practice Bulletin No. 137: Gestational diabetes mellitus. Obstetrics and gynecology, 122 (2 Pt 1), 406-416.

[7] Walker J. D. (2008). NICE guidance on diabetes in pregnancy: management of diabetes and its complications from preconception to the postnatal period. NICE clinical guideline 63. Diabetic Medicine, 25 (9), 1025-1027.

[8] McElduff, A., Cheung, N. W., McIntyre, H. D., Lagström, J. A., Oats, J. J., Ross, G. P., Simmons, D., Walters, B. N., Wein, P., \& Australasian Diabetes in Pregnancy Society (2005). The Australasian Diabetes in Pregnancy Society consensus guidelines for the management of type 1 and type 2 diabetes in relation to pregnancy. The Medical Journal of Australia, 183 (7), 373-377.

[9] Seshiah, V., Das, A. K., Balaji, V., Joshi, S. R., Parikh, M. N.,
Gupta, S., \& Diabetes in Pregnancy Study Group (2006). Gestational diabetes mellitus--guidelines. The Journal of the Association of Physicians of India, 54, 622-628.

[10] Mahalakshmi, M. M., Bhavadharini, B., Maheswari, K., Anjana, R. M., Jebarani, S., Ninov, L., Kayal, A., Malanda, B., Belton, A., Uma, R., Mohan, V., \& Unnikrishnan, R. (2016). Current practices in the diagnosis and management of gestational diabetes mellitus in India (WINGS-5). Indian Journal of Endocrinology and Metabolism, 20 (3), 364-368.

[11] Appajigol, J. S., Bellary, S. (2015). Knowledge and practices of rural family physicians and obstetricians towards gestational diabetes mellitus. Int J Community Med Public Health, 2 (2): 153-55.

[12] Utz, B., Assarag, B., Essolbi, A., Barkat, A., Delamou, A., \& De Brouwere, V. (2017). Knowledge and practice related to gestational diabetes among primary health care providers in Morocco: Potential for a defragmentation of care? Primary Care Diabetes, 11 (4): 389-396.

[13] Carballo, M., Al Wotayan, R., \& Maclean, E. C. (2016) Primary Health Care Staff Knowledge and Practices towards Gestational Diabetes Mellitus in Kuwait. Journal of Family Medicine, 3 (8): 1083.

[14] Diagnostic criteria and classification of hyperglycaemia first detected in pregnancy: a World Health Organization Guideline. (2014). Diabetes Research and Clinical Practice, 103 (3): 341363.

[15] Caissutti, C., \& Berghella, V. (2017). Scientific Evidence for Different Options for GDM Screening and Management: Controversies and Review of the Literature. BioMed Research International, 2017: 2746471.

[16] Benhalima, K., Van Crombrugge, P., Devlieger, R., Verhaeghe, J., Verhaegen, A., De Catte, L., \& Mathieu, C. (2013). Screening for pregestational and gestational diabetes in pregnancy: a survey of obstetrical centers in the northern part of Belgium. Diabetology \& Metabolic Syndrome, 5 (1): 66.

[17] Vercammen, E., Van Hoof, L., Vercammen, C., Goderis, G., \& Benhalima, K. (2020). Screening and follow-up of pregestational diabetes and gestational diabetes mellitus: A survey of primary care physicians in Belgium. Primary Care Diabetes, 14 (6): 628-632.

[18] Rayanagoudar, G., Moore, M., Zamora, J., Hanson, P., Huda, M. S., Hitman, G. A., \& Thangaratinam, S. (2015). Postpartum care of women with gestational diabetes: survey of healthcare professionals. European Journal of Obstetrics, Gynecology, and Reproductive Biology, 194: 236-240.

[19] Seshiah, V., Balaji, V., Balaji, S., Sekar, A., Sanjeevi, C. B., \& Green, A. (2005). One step screening procedure for screening and diagnosis of gestational diabetes mellitus. J Obstet Gynecol India. 55 (6): 525-29.

[20] Selim, S. (2020). Comparison of Screening tests of GDM. Gestational Diabetes Mellitus: From Diagnosis to Treatment. Nova Science Publishers, Inc 2020. 